\title{
Cross-section determination for one- and two-photon absorption of cobalt at hard-x-ray energies
}

\author{
Krzysztof Tyrała, ${ }^{1}$ Christopher Milne, ${ }^{2}$ Klaudia Wojtaszek, ${ }^{1}$ Anna Wach, ${ }^{1}$ Joanna Czapla-Masztafiak, ${ }^{1}$ \\ Wojciech M. Kwiatek, ${ }^{1}$ Yves Kayser, ${ }^{3}$ and Jakub Szlachetko, ${ }^{1, *}$ \\ ${ }^{1}$ Institute of Nuclear Physics, Polish Academy of Sciences, PL-31342 Krakow, Poland \\ ${ }^{2}$ Paul Scherrer Institute, Villigen 5232, Switzerland \\ ${ }^{3}$ Physikalisch-Technische Bundesanstalt (PTB), Abbestrasse 2-12, 10587 Berlin, Germany
}

(Received 20 February 2019; published 17 May 2019)

\begin{abstract}
For incident $\mathrm{x}$-ray energies below the core level ionization threshold, one-photon absorption (OPA) leads to off-resonant $\mathrm{x}$-ray excitations that are mediated by a virtual intermediate electronic state. In the linear regime of $\mathrm{x}$-ray interaction, this intermediate state may decay radiatively and the maximum energy of the emitted photon is given by the initial and final atomic state as well as the energy of the incident photon. In the nonlinear excitation regime, however, this intermediate state may be depleted by a second x-ray absorption process as described in the sequential two-photon absorption (TPA) mechanism. Since the same intermediate state is involved in the OPA and TPA processes, the cross-section values for OPA and TPA are interconnected. We report on the determination of cross-section values for off-resonant excitation conditions, and show how the data can be used for estimating the TPA cross section in the sequential absorption mechanism. The determined off-resonant cross sections for cobalt exhibit a Lorentz-like energy-dependent behavior allowing for an energy-dependent description of the OPA and TPA cross sections with semiempirical models. The dependence of TPA cross sections on atomic number is discussed and different scaling laws are investigated. The effect of the lifetimes of a virtual intermediate state is also studied in relation to the incidence $\mathrm{x}$-ray energy.
\end{abstract}

DOI: 10.1103/PhysRevA.99.052509

\section{INTRODUCTION}

Cross sections are important physical values that describe the probability of a process to happen under certain experimental conditions. In the case of photon-matter interaction, the cross sections are proportional to the total mass attenuation coefficient, which contains all possible interaction paths like elastic and inelastic scattering, photoionization, etc. Knowledge of the exact values of the cross sections is essential for a thorough understanding of the interaction phenomena and experimental data interpretation as well as the development of new research methods. The advent of $\mathrm{x}$-ray free-electron laser (XFEL) sources only recently opened a possibility to explore the nonlinear regime of $\mathrm{x}$ ray-matter interactions. Different reports on multiphoton interactions with a single atom within femtosecond timescales were published [1-4]. One of these nonlinear processes is the two-photon absorption mechanism in which a bound electron interacts with two separate photons within a very short time span and gets promoted to a higher energy level. In the optical regime of the electromagnetic wave spectrum two-photon absorption (TPA) has been known for years and is extensively used, for example, in two-photon microscopy $[5,6]$. In the hard-x-ray energy range, however, the TPA process has been observed only recently thanks to the use of XFELs [4,7]. This phenomenon is interesting from the point of view of both fundamental studies and applied X-ray spectroscopies. For the latter, TPA processes are of interest because of the selection rules for atomic transitions where the angular momentum of the electron can be changed by \pm 2 or 0 , unlike in the linear regime where dipole transitions are dominant in the spectral shapes (angular momentum change of \pm 1 ). The possibility of performing quadrupole-allowed spectroscopy will allow probing the quadrupole $1 s \rightarrow 3 d$ or $1 s \rightarrow$ $4 d$ transitions that are forbidden in the linear regime [4,7]. However, before real application using the TPA mechanism in $\mathrm{X}$-ray spectroscopy methods can be realized, the values of the $\mathrm{x}$-ray TPA cross sections as well as their dependence on the incident photon energy or the atomic number of the scattering material have still to be determined.

The two-photon absorption process can occur in two ways, either through simultaneous or through sequential excitation. The Hamiltonian describing both types of interaction between $\mathrm{X}$-ray radiation and the electron can be written as [7]

$$
H^{\prime}=-\frac{e}{m c} \boldsymbol{p} \cdot \boldsymbol{A}+\frac{e}{2 m c^{2}} \boldsymbol{A}^{2},
$$

where $A$ is the vector potential of x-ray radiation and $p$ is the momentum operator. Indeed, the first term of the Hamiltonian describes the sequential TPA process while the second term describes simultaneous TPA. Equation (1) was recently examined by Tamasaku et al., who came to the conclusion that the contribution of the second term is negligible [7], being a factor of two orders of magnitude lower than the $\boldsymbol{p A}$ term. This observation is consistent with recent experimental data suggesting that the TPA mechanism is purely sequential [4,7-9] at the applied XFEL X-ray fluxes and that the simultaneous absorption of two photons by one bound electron has to be considered only for extremely high x-ray fluences. 
During the sequential TPA process, the first step has to be considered as an electronic excitation to a virtual intermediate state by a photon having an energy smaller than the binding energy of the core electron. This type of excitation is also observed in the linear regime and described as off-resonant excitation or resonant Raman scattering. The sequential TPA cross sections are then described by the following equation $[4,10]$ :

$$
\sigma_{\mathrm{TPA}}=\sigma_{1} \tau_{\mathrm{vi}} \sigma_{2}
$$

where $\sigma_{1}$ is the cross section for the first excitation step, $\tau_{\mathrm{vi}}$ is the lifetime of the virtual intermediate state, and $\sigma_{2}$ describes the cross section for the second x-ray absorption process. It is apparent from Eq. (2) that the information on $\sigma_{1}$ and $\tau_{\mathrm{vi}}$ is crucial to assess the cross-section values for the TPA process $[4,8]$. The lifetime of the virtual state might be calculated for a given incident beam energy by use of the formula that connects the lifetime of the virtual intermediate state with the width of the core excited state and the incident photon energy and energy of the initial core state [11-13]:

$$
\tau_{\mathrm{vi}}=\frac{\hbar}{\sqrt{\left(E_{1 s}-E_{0}\right)^{2}+\Gamma_{1 s}^{2}}} .
$$

A schematic representation of both the off-resonant and the TPA process is shown in Fig. 1.

Up to now the off-resonant cross sections $\left(\sigma_{1}\right)$ were measured for a few elements only, using monochromatized radiation from x-ray tubes [14-16] or synchrotron radiation [17-23]. The off-resonant cross sections are usually determined by means of low-energy resolution $\mathrm{x}$-ray detectors in a fixed $45^{\circ}$ in, $-45^{\circ}$ out geometry. The low-energy-resolution measurements allow recording the entire energy range of the $\mathrm{x}$-ray emission spectrum, thus providing in principle complete information if the energy-dependent detector efficiency is accounted for and the detector response function is known. On the other hand, the application of high-energy-resolution detection allows for a more accurate determination of the measured signal shapes and positions (at the cost of lower experimental efficiency), giving thus a more detailed insight on the energy transfer of the off-resonant excitation.

\section{EXPERIMENTAL SETUP}

In the experiment, the off-resonant spectra were measured using the high-energy-resolution von Hamos spectrometer of the SuperXAS beamline at the SLS synchrotron radiation source in Switzerland [24]. Measurements were performed using a metallic cobalt sample. Cobalt is characterized by an electronic configuration having an incompletely filled $3 d$ electron shell: [Ar] $3 d^{7} 4 s^{2}$. This property makes it an interesting element with respect to eventual applications using the TPA mechanism in order to investigate the quadrupole $1 s \rightarrow 3 d$ transition. In the experimental setup a germanium diffraction (440) crystal was used to reflect the emitted radiation at the Bragg angle of $63.9^{\circ}$ around energy of the Co $K \alpha$ line. The spectra were recorded by a two-dimensional (2D) Pilatus detector [25]. During the experiment, the different incident photon energies below the $1 \mathrm{~s}$ absorption edge of cobalt were selected-from 140 to $15 \mathrm{eV}$ below the ionization threshold.
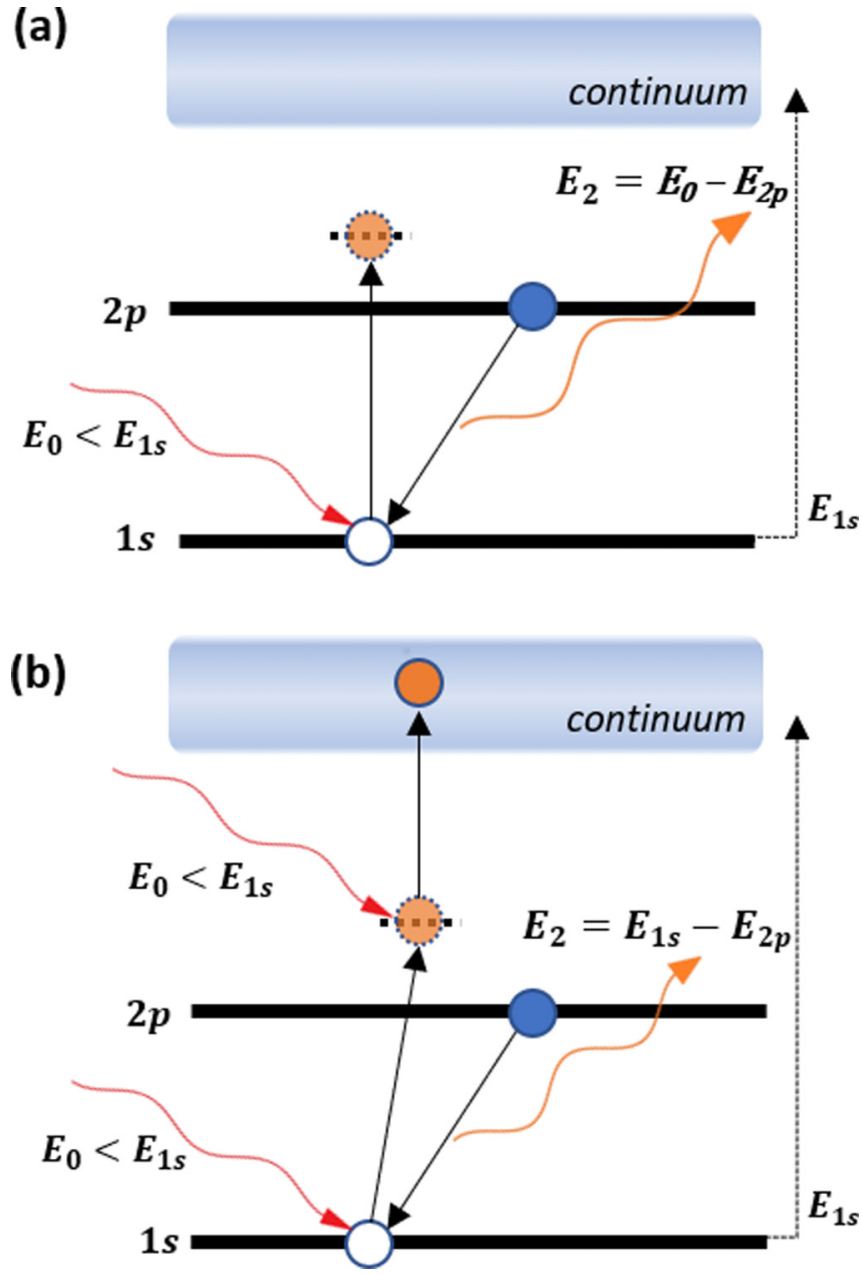

FIG. 1. Off-resonant excitation and sequential two-photon absorption are both initiated by the promotion of a core electron to an intermediate virtual state with a subfemtosecond lifetime. The energy of the incident photon energy is lower than the ionization energy of the $1 s$ shell. In the possible following radiative decay after the off-resonant excitation (a), the energy difference toward the ionization of the atom is provided at the expense of the emitted fluorescence photon for reasons of energy conservation. As a consequence, the emitted photon energy is smaller compared to the parent $K \alpha$ x-ray emission line. In the case of the two-photon process (b), the process starts also with excitation to an intermediate virtual state. However, the intermediate state is depleted by a second photon absorption process. Since the energy of each photon is at least half the absorption edge energy, the scattering atom is ionized. The radiative decay transition is then characterized by a constant photon energy equal to the energy difference between the initial and final electronic configurations of the atom.

For normalization and calibration purposes the diagram $K \alpha$ $\mathrm{x}$-ray emission signal was measured at an energy of $100 \mathrm{eV}$ above the $1 s$ ionization threshold.

\section{DATA ANALYSIS PROCEDURE}

Exemplary experimental high-energy-resolution off-resonant x-ray emission spectra are presented in Fig. 2, together with the scaled $K \alpha_{1,2}$ line which was measured at 


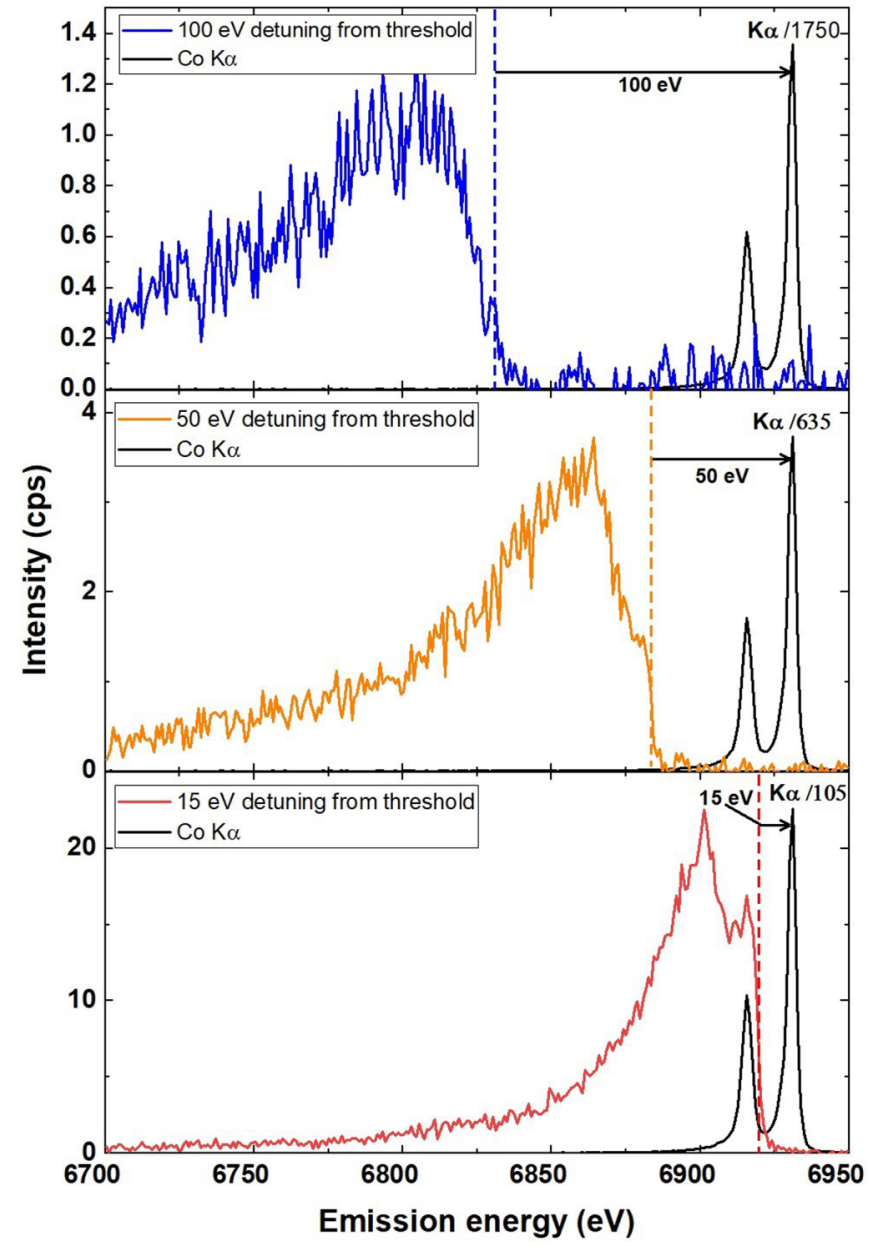

FIG. 2. High-energy-resolution off-resonant emission spectra of cobalt measured for detuning energies of 100,50 , and $15 \mathrm{eV}$ below the $K$-shell ionization threshold (top to bottom, blue, orange, and red lines). The diagram $K \alpha$ x-ray emission recorded at an incident photon energy above the ionization threshold is plotted as a smooth black line. For visualization purposes, the $K \alpha$ intensity is rescaled as marked in the inset. The vertical dotted line represents the highenergy cutoff in agreement with the energy conservation principlethe characteristic feature of the off-resonant spectra.

an incidence $\mathrm{x}$-ray energy of $100 \mathrm{eV}$ above the ionization threshold. The $K \alpha$ diagram lines are composed of two lines connected to the spin-orbit coupling of $1 / 2$ and $3 / 2$ states of the $2 p$ subshell. The intensity ratio for $K \alpha_{1,2}$ signals is equal to 0.51 as a result of the subshell transitions probabilities. The off-resonant x-ray emission spectra exhibit a broad structure with a hardly visible dual structure resulting from different spin configurations of the final state (in analogy to the $K \alpha$ diagram lines). The broad structure of the off-resonant spectra results from the excitation probability versus kinetic energy of the excited electron. As a result, the spectra are of asymmetric shape with a long, low-energy tail and characteristic high-energy cutoff, related to the energy conservation principle. The high-energy cutoff is given as the energy difference between characteristic $K \alpha_{1,2}$ emission lines and detuning energy from the ionization threshold.
From the measured x-ray emission intensities, the cross sections for off-resonant excitations may be determined. The procedure explores the fact that the cross-section values for above-threshold ionization energies are well known and may thus be employed as a direct parameter for determination of off-resonant probability excitations. Since the same setup is used for $K \alpha_{1,2}$ and off-resonant x-ray emission detection, the following formulas connecting the cross-section parameter with measured experimental intensities may be used:

$$
\begin{gathered}
I_{0} \sigma_{K \alpha} \Omega \omega_{k}=I_{K \alpha}, \\
I_{0} \sigma_{\text {off-res }} \Omega \omega_{k}=I_{\text {off-res }}, \\
\sigma_{\text {off-res }}=\frac{I_{\text {off-res }} \sigma_{K \alpha}}{I_{K \alpha}},
\end{gathered}
$$

where $I_{0}, I_{K \alpha}$, and $I_{\text {off-res }}$ are the measured intensities for incident, $K \alpha$ x-ray emission, and off-resonant radiation, respectively; $\sigma_{\text {off-res }}$ and $\sigma_{K \alpha}$ represent the cross sections for off-resonant excitation and photoionization, $\Omega$ is the solid angle, and $\omega_{k}$ is the fluorescence yield, which was assumed to be the same in both off-resonant and nonresonant excitation. A number of studies report the same shapes of x-ray absorption spectra around the ionization threshold measured with X-ray fluorescence and electron yield techniques indicating no significant deviation in the fluorescence yield value $[26,27]$. Differences in fluorescence yields around the ionization threshold are expected only for higher atomic levels (i.e., in the soft-X-ray energy range) where Auger decays involve valence electrons and multiplet effects become significant [28]. In the present case, decay of the $1 s$ core hole involves mostly either $L-K$ fluorescence or $K L L$ Auger electrons and therefore we do not expect a significant variation in fluorescence yield at incidence x-ray energies around the ionization threshold.

The determination of cross sections requires corrections for self-absorption and sample thickness on the basis of the Lambert-Beer law. Using the knowledge of the mass attenuation coefficients for the incident and the emitted photon energies, and considering the orientation of the incident beam axis, the sample surface orientation and the crystal position with respect to each, the correction factor, for both off-resonant and $K \alpha$ spectra, is given by the following equation [Eq. (7)] [29]:

$$
G=\frac{1-e^{-\sqrt{2} x \rho\left[\mu_{0}+\csc (\phi) \mu_{f}\right]}}{\rho\left[\mu_{0}+\csc (\phi) \mu_{f}\right]},
$$

where $x$ represents the sample thickness; $\rho$ is the density of given element; $\mu_{0}$ and $\mu_{f}$ are total mass attenuation coefficients for incident $\mathrm{x}$-ray energy and $K \alpha$ x-ray fluorescence energy, respectively. Finally, $\phi$ represents the Bragg angle for each emission energy. In order to cross-check the self-absorption corrections procedure the measurements were performed for two cobalt foils with different thicknesses- 6 and $25 \mu \mathrm{m}$.

The drawback of using a high-energy-resolution setup is the limited energy range that is covered. As a consequence, the spectral tail that expands toward lower emission energies is not recorded due to the finite size of the spectrometer arrangement. In order to assess the total intensity of the off-resonant 
X-ray emission signal ( $\left.I_{\text {off-res }}\right)$, an evaluation of the low-energy tail of each off-resonant spectrum is necessary. This can be achieved by fitting the theoretical function, which describes the shape of the off-resonant emission spectra to the actual, measured data. The tail of the off-resonant spectra is given by the simplified Kramers-Heisenberg formula developed by Tulkki and Aberg [30] to describe interaction of $\mathrm{x}$ rays with bound electrons around threshold energies:

$$
\frac{d \sigma\left(E_{1}\right)}{d E_{1}}=A \int_{0}^{\infty} \frac{E_{2}}{E_{1}} \frac{\left(E_{1 s}-E_{2 p j}\right) g_{2 p j}\left(E_{1 s}+E\right)}{\left(E_{1 s}+E-E_{1}\right)^{2}+\Gamma_{1 s}^{2} / 4} \frac{\Gamma_{2 p j}^{2} / 2}{\left(E_{1}-E_{2 p j}-E-E_{2}\right)^{2}+\Gamma_{2 p j}^{2} / 4} d E,
$$

where $E_{1}$ is the incident and $E_{2}$ is the fluorescent photon energy, $E$ represents the energy above the Fermi level of the excited electron, $j$ corresponds to the total angular momentum of the final state ( $1 / 2$ or $3 / 2$ in our case), and $A$ is a normalization constant taking the solid angle and detection efficiency of the experimental setup into account. The above equation was used to calculate the X-ray emission tail, which was fitted to the recorded off-resonant data in order to acquire the information about the intensity outside the energy range monitored by the spectrometer. One example of this calculation is shown in Fig. 3.

After fitting, the theoretical function was integrated and the area of the region outside the range covered by the spectrometer was added to the measured intensity. This approach allowed obtaining the total intensity of the off-resonant spectrum, and hence for the off-resonant cross-section determination. The relative contribution of the intensity outside the spectrometer's energy window is about $40 \%$ of the total intensity at the lowest incident photon energy and almost $0 \%$

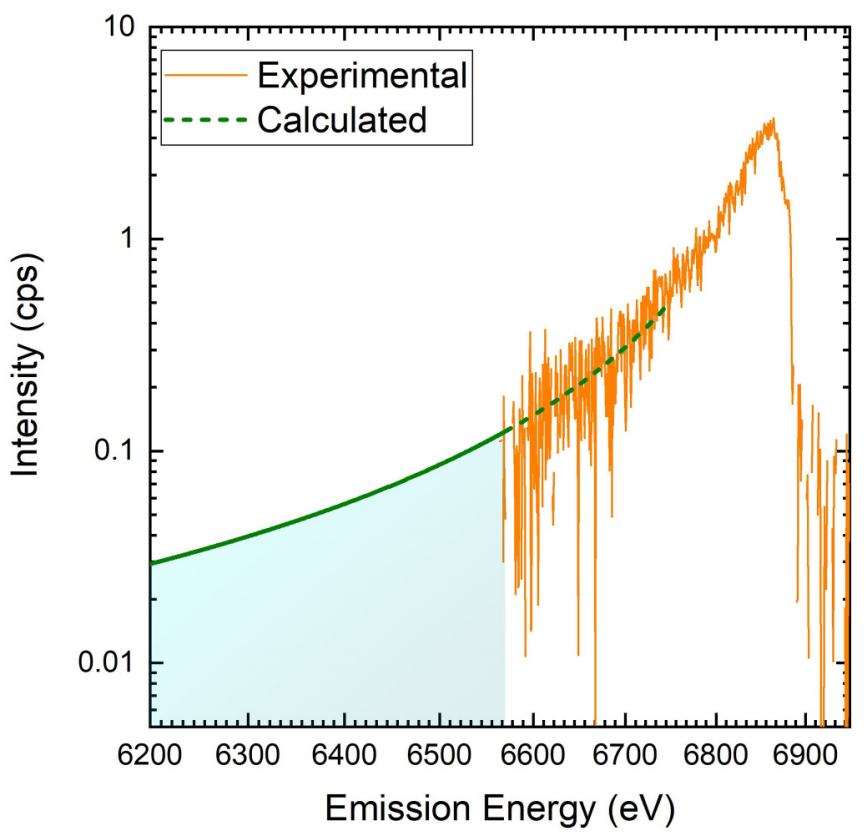

FIG. 3. Example of the measured off-resonant spectrum for an incident photon energy of $7569 \mathrm{eV}$ (orange line) and plots of the calculated and fitted low-energy Lorentz-like tail (green dashed and solid lines) using the Kramers-Heisenberg theorem. The dashed part of the tail represents the fitting range of the curve, and the solid line with shaded area below shows the values that were extrapolated in order to determine the total intensity of the off-resonant spectrum. for incident photon energies in the vicinity of the ionization threshold.

The measured off-resonant x-ray emission intensities and the calculated off-resonant cross-section values for both cobalt samples are plotted in Fig. 4. Panel (a) represents the total intensities of the spectra for both 6- and 25- $\mu \mathrm{m}$ thickness of samples, showing the difference due to self-absorption as well as the energy dependences. Panel (b) shows the crosssection values determined for both thicknesses, calculated using self-absorption corrected spectra and Eq. (6). As shown, the applied correction eliminates the difference between the measured intensities for the two samples, thus validating the self-absorption correction procedure. The data agree with each other in a range of $\pm 10 \%$.

Finally, the average value between both sample thicknesses was used to determine the cross section for off-resonant excitation. The result is plotted in Fig. 5(a). The experimental data were fitted with a Lorentz-like function that can be derived by integration of Eq. (8) over the emission energy $E_{2}$.

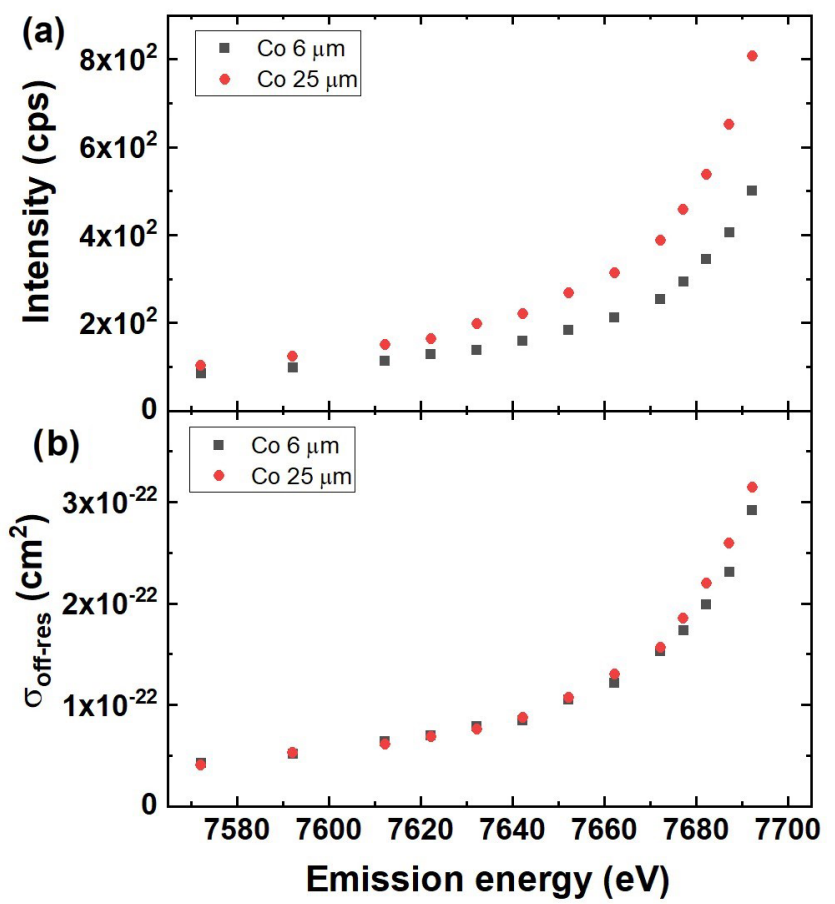

FIG. 4. Measured intensity (a), and determined cross-section values (b) considering self-absorption corrections for 6- and 25$\mu \mathrm{m}$ cobalt samples. The difference between the values of both thicknesses is in the range of $10 \%$ of the cross-section value at the different incident photon energies. 


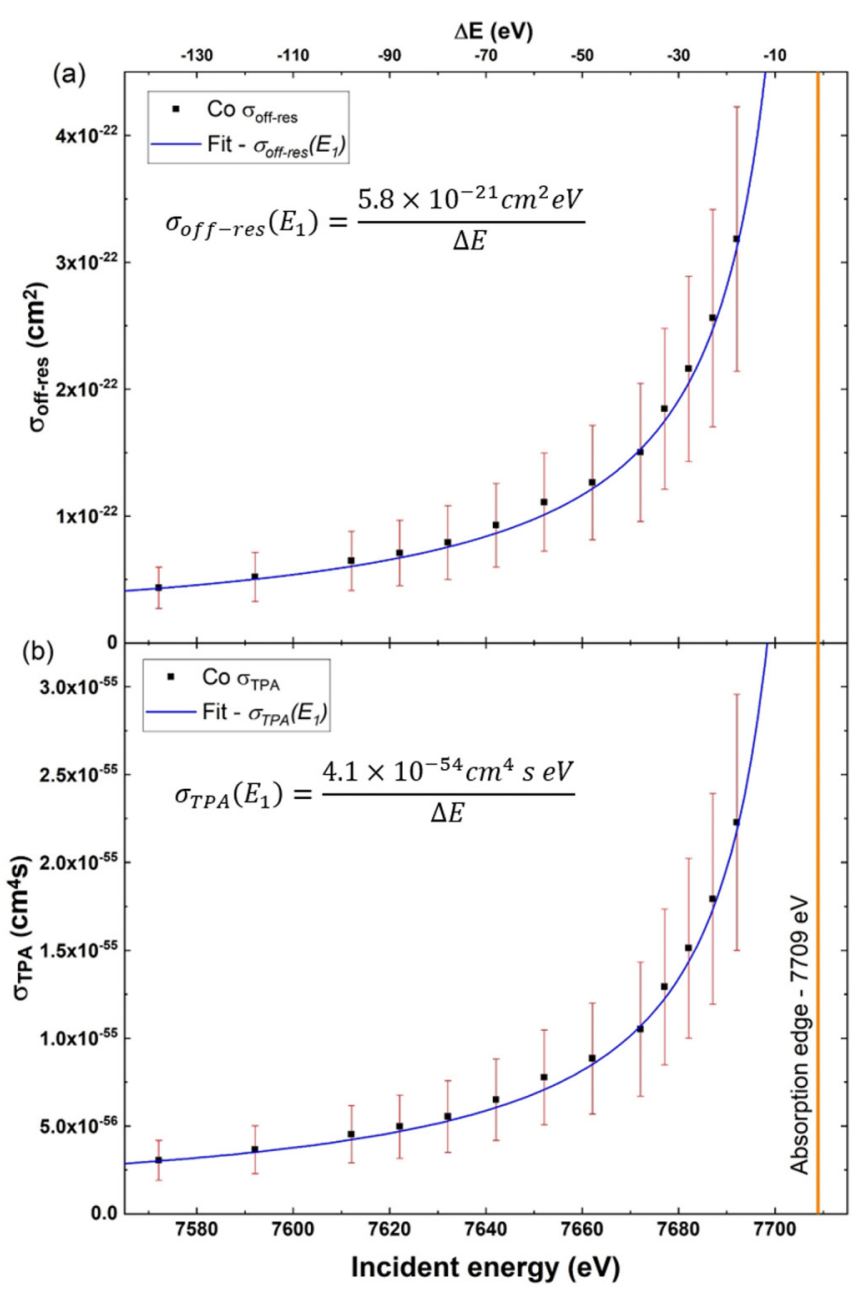

FIG. 5. Off-resonant cross-section value for $\mathrm{Co}$, in $\mathrm{cm}^{2}$ (a) and the TPA cross-section values in $\mathrm{cm}^{4} \mathrm{~s}$ (b). The black squares represent the calculated data; the blue solid lines correspond to the fit of the Lorentz function data using the equation inserted in graphs; the orange vertical line corresponds to the absorption edge for Co $(7709 \mathrm{eV})$.

The parameter $\Delta E$ represents the detuning energy from the ionization threshold and is equal to $E_{1 s}-E_{1}$. As shown, the fitted function depicts very well the experimental data points and may thus be used for an empirical determination of the dependence of the off-resonant cross section over a broad incident energy range $E_{1}$. For an incident energy range between 7550 and $7690 \mathrm{eV}$ the cross-section values for off-resonant excitation vary between $4.0 \times 10^{-23}$ and $3.0 \times 10^{-22} \mathrm{~cm}^{2}$. The relative values of the calculated cross-section error bars are about $35 \%$, and take into consideration the uncertainties on the used cross sections and attenuation coefficients in the self-absorption correction as well as the uncertainty on sample thickness and statistical errors in the data analysis (including the uncertainty of the fitted part of the off-resonant spectra). The parameter used in the fit function was found to be $5.8 \times 10^{-21} \mathrm{~cm}^{2} \mathrm{eV}$ and represents the off-resonant excitation cross section in proximity to the absorption edge $(\Delta E \leqslant$ $1 \mathrm{eV})$. Using the same function it is possible to estimate the cross-section value at maximum detuning energy, given by the energy conservation principle included in the second term of the equation $E_{1}-E_{2 p j}-E-E_{2}$. In the case of cobalt, the maximum experimental incident photon energy detuning for the final state of $2 p_{1 / 2}$ is equal to $6915.8 \mathrm{eV}$, for which the fitted function gives the cross-section value of $\sim 8.4 \times$ $10^{-25} \mathrm{~cm}^{2}$, i.e., four orders of magnitude lower than in the vicinity of the $1 s$ ionization threshold. Since we did not find any literature data for the Co off-resonant excitation cross sections, we compared the obtained results with the crosssection values for the neighbor elements- $\mathrm{Fe}$ and $\mathrm{Cu}$. At 50 $\mathrm{eV}$ energy detuning the determined Co cross-section data is equal to $1.16 \times 10^{-22} \mathrm{~cm}^{2}$, and the literature values for $\mathrm{Fe}$ and $\mathrm{Cu}$ are $1.54 \times 10^{-22}$ and $1.22 \times 10^{-22} \mathrm{~cm}^{2}$, respectively $[16,17]$. Assuming the $Z^{-2}$ dependence of the cross-section values, as for the photoionization above the $K$-shell ionization threshold, the expected value for $\mathrm{Co}$, derived based on $\mathrm{Fe}$ and $\mathrm{Cu}$ data, is equal to $1.41 \times 10^{-22} \mathrm{~cm}^{2}$, which is within a $20 \%$ difference of our experimental data.

The so-obtained cross sections for off-resonant excitation were then used for estimating the two-photon absorption cross section [Eq. (2)]. Recent reports regarding the experiments performed with XFEL suggested that the sequential TPA cross sections are directly connected to off-resonant excitation via a scaling factor [4]. Based on experimental data, the scaling factor describing the relation between one- and two-photon absorption cross sections was estimated to be $7.0 \times 10^{-34} \mathrm{~cm}^{2} \mathrm{~s}[4]$. This factor represents the ratio between multiphoton absorption cross sections, similarly to the powerlaw value at laser wavelengths. In the simplest assumption, the same coefficient may be used for calculating the twophoton absorption cross sections for cobalt. We should note here that the assumption is only valid for the sequential TPA mechanism in which the off-resonant excitation is regarded as the first absorption event.

The resulting cross section for the TPA process for cobalt as a function of incidence energy is plotted in Fig. 5(b). Assuming a direct connection between the OPA and TPA cross sections, the data can also be fitted with a Lorenz-like shaped function starting from Eq. (8). Again $\Delta E$ represents the incident photon energy detuning with respect to the onephoton absorption edge for cobalt. The value in the numerator is the fitted parameter with a value of $4.1 \times 10^{-54} \mathrm{~cm}^{4} \mathrm{~s} \mathrm{eV}$. This parameter, can be, analogously like in the case of OPA, understood as a TPA cross-section value in the vicinity of the $1 s$ ionization threshold. As shown, the TPA excitation probability is driven by the probability of the first absorption event. In contrast to off-resonant excitation conditions, in the two-photon process, the maximum photon energy detuning is determined by half of the absorption edge energy, $3854.5 \mathrm{eV}$ for cobalt, and we estimate a TPA cross-section value at this energy to be $\sim 1.1 \times 10^{-57} \mathrm{~cm}^{4} \mathrm{~s}$. This estimation does not include eventual resonant effects for the second absorption event in the sequential process [31].

Since the cross sections for OPA and TPA processes have different units ( $\mathrm{cm}^{2}$ for OPA and $\mathrm{cm}^{4} \mathrm{~s}$ for TPA) the estimation of expected intensities requires a determination of the rates for both processes. At typical experimental conditions provided at XFEL facilities, incidence $\mathrm{x}$-ray intensities in terms of photons per pulse vary between $10^{11}$ and $10^{12}$ and repetition rates are in the range of a few tens of hertz. Considering incidence $\mathrm{x}$-ray pulse durations of $30 \mathrm{fs}$ and a $10 \times 10 \mu \mathrm{m}^{2}$ 


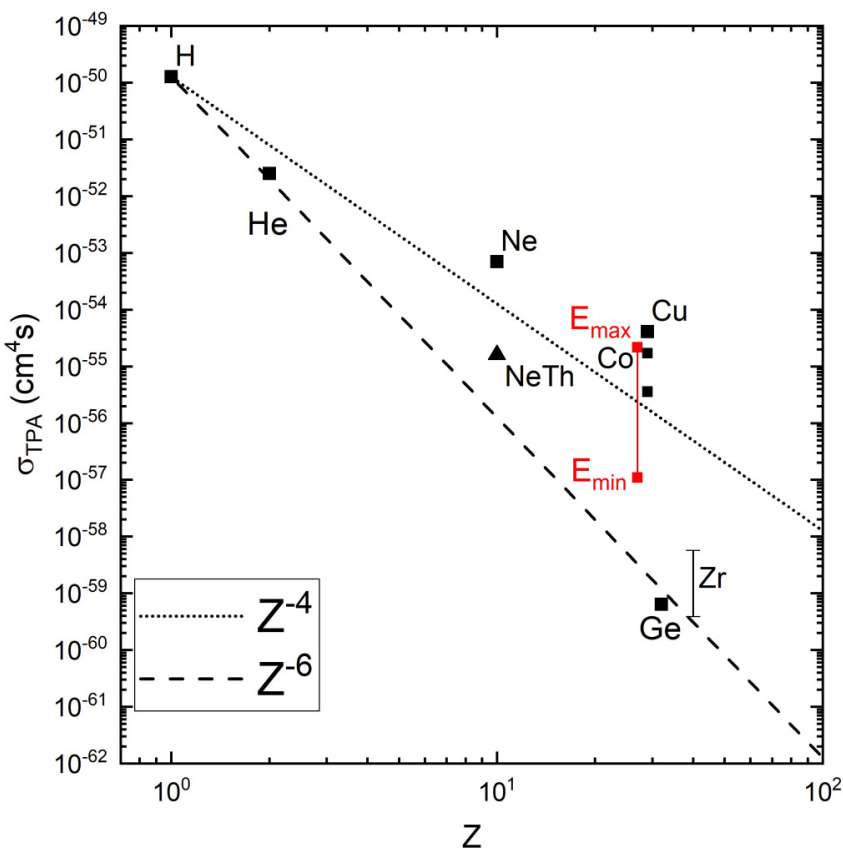

FIG. 6. The available TPA cross-section values plotted versus the atomic number of the elements. The dotted line is the $Z^{-4}$ and the dashed line is the $Z^{-6}$ scaling law, respectively. The estimated values of Co cross sections are presented from the minimum to the maximum detuning of incident energy. The data for $\mathrm{H}$ are taken from [32] and for He from [33]; the Ne data were obtained at energy being detuned by $86 \mathrm{eV}$ from $\mathrm{Ne}^{8+} K$-shell ionization threshold [34], and the theoretical value (triangle) is taken from [35]; the $\mathrm{Cu}$ data provided in [4] were obtained by using energies ranging from 12 to $150 \mathrm{eV}$ below the $K$-shell ionization threshold. Cross sections for $\mathrm{Ge}$ were established at $5.5 \mathrm{keV}$ detuning [7]; finally the upper data value for $\mathrm{Zr}$ corresponds to $9.1 \mathrm{keV}$ energy detuning [9].

beam focusing of the average flux value of $1 \times 10^{14}$ photons $/ \mathrm{s}$, a fluence of about $10^{31}$ photons $/\left(\mathrm{cm}^{2} \mathrm{~s}\right)$ is obtained. The rates for OPA and TPA processes can be expressed as a $\sigma_{\mathrm{OPA}} \times I$ and $\sigma_{\mathrm{TPA}} \times I^{2}$, respectively. Using the cross-section values for one- and two-photon absorption, at $50 \mathrm{eV}$ detuning from the $1 s$ ionization threshold $\left(1.16 \times 10^{-22} \mathrm{~cm}^{2}\right.$ and $8.2 \times$ $10^{-56} \mathrm{~cm}^{4} \mathrm{~s}$, respectively), one may calculate rate values of $2 \times 10^{9} \mathrm{~s}^{-1}$ for OPA and $2 \times 10^{7} \mathrm{~s}^{-1}$ for TPA signals. The difference between the OPA and TPA rates at assumed incidence $\mathrm{x}$-ray conditions is thus around a factor of $10^{2}$, which directly corresponds to the strength differences between the signals expected at the described conditions.

Based on the derived cross sections for Co and available experimental and theoretical literature data $[4,7,9,31-35]$ we evaluated the dependence of the TPA cross section as a function of the atomic number. The result is presented in Fig. 6 together with the scaling laws assuming either a $Z^{-4}$ dependence resulting from the Kramers-Heisenberg relation [4] or a $Z^{-6}$ dependence as expected for hydrogenlike atoms [36]. The cross sections for the measured Co data are presented for the energy range from $3855 \mathrm{eV}$, which corresponds to the maximum detuning for the TPA process (i.e., half of the $1 \mathrm{~s}$ threshold value), to the value of $7690 \mathrm{eV}$ that corresponds to a detuning equal to about $15 \Gamma_{1 s}$ (i.e., the data point measured closest to the $1 s$ threshold). A general conclusion drawn from Fig. 6 is that the cross sections for $\mathrm{Ne}$ and $\mathrm{Cu}$ are closer to the $Z^{-4}$ dependence, while $\mathrm{Ge}$ and $\mathrm{Zr}$ data are properly described by the $Z^{-6}$ function. There are two important factors that may explain the observed trends.

The first factor that should be considered when comparing the data is the incident photon energy for which the actual TPA cross-section values were determined. The $\mathrm{Ne}$ and $\mathrm{Cu}$ TPA cross sections were measured relatively close to the $K$-shell absorption edge, i.e., $86 \mathrm{eV}$ below the threshold for $\mathrm{Ne}$ and $12-150 \mathrm{eV}$ below the ionization threshold for $\mathrm{Cu}$, respectively. In contrast, the $\mathrm{Ge}$ and $\mathrm{Zr}$ data were measured at large detuning energies that were close to the half value of the single ionization threshold. These differences suggest the importance of the detuning energy value for the TPA process and point to a significant contribution of the virtual intermediate state lifetime on the TPA cross-section values. Indeed, as indicated by Eq. (3), the lifetime of the intermediate state will strongly depend on the detuning energy from the ionization threshold and may be expressed as a $Z^{-2}$ dependence, which fits into the gap between the $Z^{-4}$ and $Z^{-6}$ scaling laws. The $Z^{-2}$ dependence results from Eq. (3), specifically from the $Z$ dependence on the core-hole width $\Gamma$ [37]. This observation is also confirmed by the Co TPA cross section determined in the present work. As shown in Fig. 6, for incidence x-ray energies close to the $1 s$ threshold the TPA cross sections are expected to follow a $Z^{-4}$ dependence and decrease toward a $Z^{-6}$ scaling at larger detuning energies.

Secondly, we should emphasize that the $\mathrm{Ne}$ and $\mathrm{Cu}$ data were measured using the Linac Coherent Light Source (LCLS) machine while the $\mathrm{Ge}$ and $\mathrm{Zr}$ data were obtained with the SACLA XFEL. It should be noted that the SACLA operates with shorter pulses ( $2-8 \mathrm{fs}$ ) while the LCLS delivers significantly longer X-ray pulses (30-80 fs). Moreover, because of the self-amplified spontaneous emission (SASE) mode that drives the XFEL's operation, spiky features characterize the temporal spectral shape of the delivered $\mathrm{x}$-ray pulses. The $\mathrm{x}$-ray pulse spikiness is described by the coherence time that at the limit for the LCLS machine [38] is expected to be in the timescale of core-hole lifetimes, e.g., $0.15 \mathrm{fs}$ [37] in the case of the $1 s$ hole of cobalt, while the minimum coherence time of the SACLA machine is reported to be even lower than $0.1 \mathrm{fs}$ [39]. This effect results in an amplification of the nonlinear process by a factor of 2 , due to the maximum value of the degree of second-order coherence-the parameter that might be used to describe the spiky structure of SASE pulses at XFELs [40,41]. The enhancement factor of 2 is expected for both $\boldsymbol{p} \cdot \boldsymbol{A}$ (sequential) and $\mathbf{A}^{2}$ (simultaneous) mediated TPA processes $[41,42]$ in cases when the coherence time of the incident XFEL pulse is on the order (or shorter) than the lifetime of the intermediate states.

\section{SUMMARY}

Off-resonant excitation $\mathrm{x}$-ray emission spectroscopy on cobalt was performed for incident energy detuning factors ranging from 140 to $15 \mathrm{eV}$ below the $K$-shell ionization threshold. The obtained data were interpreted on the basis of Kramers-Heisenberg formalism in order to take into account the low-energy tail of the off-resonant spectra. The 
determined cross-section values for off-resonant excitation have a Lorentz-like shape with the normalization factor of the fitted function equal to $5.8 \times 10^{-21} \mathrm{~cm}^{2} \mathrm{~s} \mathrm{eV}$. Considering the off-resonant process as the first step of sequential two-photon absorption, the TPA cross sections can be estimated. From the experimental data, a TPA cross section of $\sim 1.1 \times 10^{-57} \mathrm{~cm}^{4} \mathrm{~s}$ was determined close to the lowest possible energy for the two-photon absorption process. Based on our calculations and literature data, we discussed the $Z$ dependence of the twophoton absorption cross sections. The data for low-energy detuning suggest the $Z^{-4}$ dependence, while the measurements performed close to the maximum energy detuning (i.e., half of the single ionization threshold) seem to exhibit the $Z^{-6}$ dependence. This difference might be explained as a result of the different lifetimes of the virtual intermediate state, that is strongly related with the energy detuning, and can be described as a $Z^{-2}$ relation that connects both of the discussed $Z$ dependences for TPA cross sections.

\section{ACKNOWLEDGMENT}

The authors would like to acknowledge the National Science Center (NCN, Poland) for partial support under Grants No. 2017/27/B/ST2/01890 and No. 2015/19/B/ST2/00931.
[1] M. Beye, S. Schreck, F. Sorgenfrei, C. Trabant, N. Pontius, C. Schüßler-Langeheine, W. Wurth, and A. Föhlisch, Nature 501, 191 (2013).

[2] S. M. Vinko, O. Ciricosta, B. I. Cho, K. Engelhorn, H.-K. Chung, C. R. D. Brown, T. Burian, J. Chalupský, R. W. Falcone, C. Graves, V. Hájková, A. Higginbotham, L. Juha, J. Krzywinski, H. J. Lee, M. Messerschmidt, C. D. Murphy, Y. Ping, A. Scherz, W. Schlotter et al., Nature 482, 59 (2012).

[3] B. Nagler, U. Zastrau, R. R. Fäustlin, S. M. Vinko, T. Whitcher, A. J. Nelson, R. Sobierajski, J. Krzywinski, J. Chalupsky, E. Abreu, S. Bajt, T. Bornath, T. Burian, H. Chapman, J. Cihelka, T. Döppner, S. Düsterer, T. Dzelzainis, M. Fajardo, E. Förster et al., Nat. Phys. 5, 693 (2009).

[4] J. Szlachetko, J. Hoszowska, J.-C. Dousse, M. Nachtegaal, W. Błachucki, Y. Kayser, J. Sà, M. Messerschmidt, S. Boutet, G. J. Williams, C. David, G. Smolentsev, J. A. van Bokhoven, B. D. Patterson, T. J. Penfold, G. Knopp, M. Pajek, R. Abela, and C. J. Milne, Sci. Rep. 6, 33292 (2016).

[5] M. Göppert-Mayer, Ann. Phys. 401, 273 (1931).

[6] W. Denk, J. H. Strickler, and W. W. Webb, Science 248, 73 (1990).

[7] K. Tamasaku, E. Shigemasa, Y. Inubushi, T. Katayama, K. Sawada, H. Yumoto, H. Ohashi, H. Mimura, M. Yabashi, K. Yamauchi, and T. Ishikawa, Nat. Photonics 8, 313 (2014).

[8] K. Tyrała, K. Wojtaszek, M. Pajek, Y. Kayser, C. Milne, J. Sá, and J. Szlachetko, Appl. Sci. 7, 653 (2017).

[9] S. Ghimire, M. Fuchs, J. Hastings, S. C. Herrmann, Y. Inubushi, J. Pines, S. Shwartz, M. Yabashi, and D. A. Reis, Phys. Rev. A 94, 043418 (2016).

[10] P. Lambropoulos and X. Tang, J. Opt. Soc. Am. B 4, 821 (1987).

[11] F. Gel'mukhanov, P. Sałek, T. Privalov, and H. Agren, Phys. Rev. A 59, 380 (1999).

[12] R. Feifel, A. Baev, F. Gel'mukhanov, H. Ågren, M. N. Piancastelli, M. Andersson, G. Öhrwall, C. Miron, M. Meyer, S. L. Sorensen, A. Naves de Brito, O. Björneholm, L. Karlsson, and S. Svensson, Phys. Rev. A 69, 022707 (2004).

[13] F. Gel'mukhanov and H. Ågren, Phys. Rev. A 54, 3960 (1996).

[14] C. J. Sparks, Phys. Rev. Lett. 33, 262 (1974).

[15] P. Suortti, Phys. Status Solidi 91, 657 (2018).

[16] K. Hamalainen, S. Manninen, P. Suortti, S. P. Collins, M. J. Cooper, and D. Laundy, J. Phys.: Condens. Matter 1, 5955 (1989).

[17] H. J. Sánchez, M. C. Valentinuzzi, and C. Pérez, J. Phys. B: At., Mol. Opt. Phys. 39, 4317 (2006).
[18] J. P. Briand, D. Girard, V. O. Kostroun, P. Chevalier, K. Wohrer, and J. P. Mossé, Phys. Rev. Lett. 46, 1625 (1981).

[19] S. Manninen, P. Suortti, M. J. Cooper, J. Chomilier, and G. Loupias, Phys. Rev. B 34, 8351 (1986).

[20] J. Szlachetko, J.-C. Dousse, J. Hoszowska, M. Pajek, R. Barrett, M. Berset, K. Fennane, A. Kubala-Kukus, and M. Szlachetko, Phys. Rev. Lett. 97, 073001 (2006).

[21] M. A. MacDonald, S. H. Southworth, J. C. Levin, A. Henins, R. D. Deslattes, T. LeBrun, Y. Azuma, P. L. Cowan, and B. A. Karlin, Phys. Rev. A 51, 3598 (1995).

[22] A. G. Karydas, Nucl. Instrum. Methods Phys. Res., Sect. B 189, 43 (2002)

[23] M. Žitnik, M. Kavčič, K. Bučar, A. Mihelič, M. Štuhec, J. Kokalj, and J. Szlachetko, Phys. Rev. A 76, 032506 (2007).

[24] J. Szlachetko, M. Nachtegaal, E. de Boni, M. Willimann, O. Safonova, J. Sa, G. Smolentsev, M. Szlachetko, J. A. van Bokhoven, J.-Cl. Dousse, J. Hoszowska, Y. Kayser, P. Jagodzinski, A. Bergamaschi, B. Schmitt, C. David, and A. Lücke, Rev. Sci. Instrum. 83, 103105 (2012).

[25] P. Kraft, A. Bergamaschi, C. Broennimann, R. Dinapoli, E. F. Eikenberry, B. Henrich, I. Johnson, A. Mozzanica, C. M. Schlepütz, P. R. Willmott, and B. Schmitt, J. Synchrotron Radiat. 16, 368 (2009).

[26] A. Erbil, G. S. Cargill, R. Frahm, and R. F. Boehme, Phys. Rev. B 37, 2450 (1988).

[27] T. Guo and M. L. denBoer, Phys. Rev. B 31, 6233 (1985).

[28] F. M. F. de Groot, M. A. Arrio, P. Sainctavit, C. Cartier, and C. T. Chen, Solid State Commun. 92, 991 (1994).

[29] C. H. Booth and F. Bridges, Phys. Scr. 2005, 202 (2005).

[30] J. Tulkki and T. Aberg, J. Phys. B: At. Mol. Phys. 15, L435 (1982).

[31] K. Tamasaku, E. Shigemasa, Y. Inubushi, I. Inoue, T. Osaka, T. Katayama, M. Yabashi, A. Koide, T. Yokoyama, and T. Ishikawa, Phys. Rev. Lett. 121, 083901 (2018).

[32] F. T. Chan and C. L. Tang, Phys. Rev. 185, 42 (1969).

[33] T. Sato, A. Iwasaki, K. Ishibashi, T. Okino, K. Yamanouchi, J. Adachi, A. Yagishita, H. Yazawa, F. Kannari, M. Aoyma, K. Yamakawa, K. Midorikawa, H. Nakano, M. Yabashi, M. Nagasono, A. Higashiya, and T. Ishikawa, J. Phys. B: At., Mol. Opt. Phys. 44, 161001 (2011).

[34] G. Doumy, C. Roedig, S.-K. Son, C. I. Blaga, A. D. DiChiara, R. Santra, N. Berrah, C. Bostedt, J. D. Bozek, P. H. Bucksbaum, 
J. P. Cryan, L. Fang, S. Ghimire, J. M. Glownia, M. Hoener, E. P. Kanter, B. Krässig, M. Kuebel, M. Messerschmidt, G. G. Paulus et al., Phys. Rev. Lett. 106, 083002 (2011).

[35] S. A. Novikov and A. N. Hopersky, J. Phys. B: At., Mol. Opt. Phys. 34, 4857 (2001).

[36] W. Zernik, Phys. Rev. 135, A51 (1964).

[37] J. L. Campbell and T. Papp, At. Data Nucl. Data Tables 77, 1 (2001).

[38] P. Emma, K. Bane, M. Cornacchia, Z. Huang, H. Schlarb, G. Stupakov, and D. Walz, Phys. Rev. Lett. 92, 074801 (2004).
[39] F. Lehmkühler, C. Gutt, B. Fischer, M. A. Schroer, M. Sikorski, S. Song, W. Roseker, J. Glownia, M. Chollet, S. Nelson, K. Tono, T. Katayama, M. Yabashi, T. Ishikawa, A. Robert, and G. Grübel, Sci. Rep. 4, 5234 (2014).

[40] K. Tamasaku, M. Nagasono, H. Iwayama, E. Shigemasa, Y. Inubushi, T. Tanaka, K. Tono, T. Togashi, T. Sato, T. Katayama, T. Kameshima, T. Hatsui, M. Yabashi, and T. Ishikawa, Phys. Rev. Lett. 111, 043001 (2013).

[41] H. R. Varma, M. F. Ciappina, N. Rohringer, and R. Santra, Phys. Rev. A 80, 053424 (2009).

[42] N. Rohringer and R. Santra, Phys. Rev. A 76, 033416 (2007). 